\title{
Hybrid quasistatic analysis for multilayer coplanar lines
}

\author{
C.-N. Chang \\ Y.-C. Wong \\ C.H. Chen
}

Indexing terms: Waveguides, Transmission lines

\begin{abstract}
A new quasistatic analysis of a coplanar waveguiding structure with multidielectric layers is presented, using a hybrid approach that combines the Galerkin finite-element method and the conformal-mapping technique. In the study, Wen's mapping function is adopted, which not only transforms the infinity problem domain into a finite region, but also overcomes the field singularity difficulty around the strip edges. Numerical results for effective dielectric constants, characteristic impedances, charge distributions and field distributions for various multilayer coplanar line structures are presented and the magnetic wall assumption over the slot is also investigated.
\end{abstract}

\section{Introduction}

Accurate prediction of effective dielectric constants and characteristic impedances of transmission lines, such as microstrip lines and coplanar waveguides, is essential to the design of a microwave integrated circuit. Recently, the coplanar waveguiding structure has received increasing attention in monolithic microwave integrated circuits [1-3], mainly owing to its easy adaptation to external devices. With all conductor electrodes on the same side of the substrate, the coplanar structure is easily adopted to external shunt and series elements. It is also suitable for accommodating MESFETs and other three-terminal devices due to the existence of three separate conductors in the structure. Although the dispersive characteristics of coplanar structures are indispensable in the higher microwave spectrum, the quasistatic parameters are still useful in lower frequency regimes in which the cross-sectional dimensions of the structures are much smaller than the wavelength.

To take account of the infinite extension of fields in a coplanar line, a series of quasistatic analyses have been reported, using the conformal-mapping technique [4-10]. In 1969, Wen [4] carried out such an analysis for a coplanar waveguide with an infinitely thick substrate. By a modification of Wen's approach, David et al. [5] later took the finite thickness of the substrate into account. In 1980, Veyres and Hanna [6] obtained closed-form expressions for the characteristic impedance and effective dielectric constant of coplanar lines, based on a judicious

Paper 8016H (E12), first received 29th August 1990 and in revised form 5th February 1991

The authors are with the Department of Electrical Engineering, National Taiwan University, Taipei 10764, Taiwan, Republic of China

IEE PROCEEDINGS-H, Vol. 138, No. 4, AUGUST 1991 treatment of the line capacitance between the centre strip and the two ground strips. From 1981 to 1987, several quasistatic analyses using different conformal mapping functions [7-10] were proposed to investigate the effects of finite extent ground planes, conductor backing, upper shielding and asymmetrical apertures. Although the above analyses have considered the infinite extension of fields due to an open coplanar structure, the assumption of magnetic walls over the two slots is made in all previous analyses. Also, these analyses are difficult to evaluate the charge distribution over the signal strip and the field pattern of coplanar waveguides. Other quasistatic methods proposed for a study of coplanar waveguides were the stationary formula $[11,12]$ and the finitedifference method [13].

In addition to the substrate, dielectric films or layers are sometimes introduced over the conductor parts of coplanar waveguide in practice. These added dielectric layers may provide additional means of adjusting the transmission line characteristics. To address the effects of multidielectric layers, the general coplanar line structure shown in Fig. 1 also needs careful investigation. A new quasistatic hybrid approach for the open multilayer coplanar line is presented here, without the assumption of magnetic wall over slots. This novel approach combines both advantages of finite-element method and conformal-mapping technique. By suitably adopting a mapping function, the difficulties associated with infinite field extension and field singularity near conductor edges may be properly handled, instead of using the infinite elements and singular elements [14]. In this study, the characteristic parameters of a coplanar waveguide with multidielectric layers are carefully examined. Also investigated are the field over slots and the charge distribution over signal strip.

\section{Hybrid approach}

The open coplanar line structure with multidielectric layers (isotropic and lossless) is shown in Fig. 1. A metallic signal strip (assuming zero thickness) of width $2 a$ and two conducting ground planes (also of zero thickness) of separation $2 b$ are placed in the plane $\left(y^{\prime}=0\right)$ between two dielectric layers. Above and below the conductors are $n$ upper and $m$ lower homogeneous dielectric layers of permittivities $\tilde{\varepsilon}_{k}=\varepsilon_{0} \tilde{\varepsilon}_{r k}(k=1,2, \ldots, n)$ and $\varepsilon_{k}=\varepsilon_{0} \varepsilon_{r k}$ $(k=1,2, \ldots, m)$, respectively. By an appropriate conformal mapping function, the original infinite domain (Fig. 1 ) is first transformed into a finite image domain (Fig. 3) 
and the problem is then solved in this image domain by the Galerkin finite-element method.

\subsection{Conformal mapping}

It is well known that the upper (or lower) half $z^{\prime}$-plane in

Fig. $1\left(z^{\prime}=x^{\prime}+j y^{\prime}\right)$ may be conformally transformed into separate surfaces and the two neighbouring points $B_{1}^{\prime}$ and $B_{2}^{\prime}$ (in Fig. 1) are still mapped into the two neighbouring points $B_{1}$ and $B_{2}$ in the image plane (Fig. 2). Also from symmetric consideration, one may put a magnetic wall on $x^{\prime}=0$ and only the right half plane $x^{\prime} \geqslant 0$ (Fig. 1) needs consideration in the analysis. Therefore, in

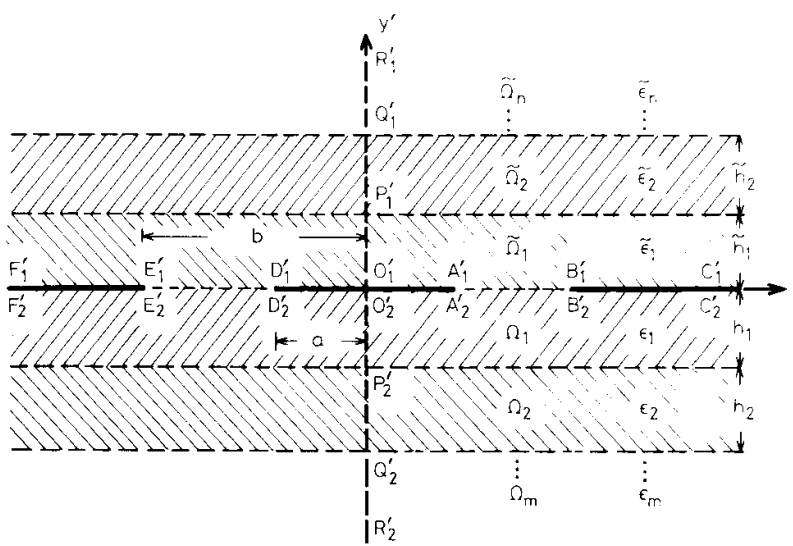

Fig. 1 Geometry of multilayer coplanar line and original domain (whole $z^{\prime}$-plane, $z^{\prime}=x^{\prime}+j y^{\prime}$ )

the interior of the rectangular region $(a)+(b)$ (or $\left(c_{1}\right)$ $\left.+\left(d_{1}\right)\right)$ with width $2 K(=2 K(k))$ and height $K^{\prime}\left(=K\left(k^{\prime}\right)\right)$ in the $z$-plane (Fig. 2, $z=x+j y$ ) by the mapping function [4]

$$
z^{\prime}=a \operatorname{sn}(z, k)
$$

where $\operatorname{sn}(z, k)$ is the sine elliptic function, $K(k)$ is the complete elliptic integral of the first kind [15], $k=a / b$ and $k^{\prime}=\sqrt{ }\left(1-k^{2}\right)$.

Owing to the double period properties of the complex sine elliptic function [16], the same mapping function (eqn. 1) may transform the whole $z^{\prime}$-plane (original domain) in Fig. 1 into either the rectangular region $(a)$ $+(b)+\left(c_{1}\right)+\left(d_{1}\right)$ or the region $(a)+(b)+(c)+(d)$ in the complex $z$-plane (Fig. 2). Hereafter, the latter rectangular region $(a)+(b)+(c)+(d)$ will be chosen as the image domain. By this choice, the upper and lower surfaces of all conducting strips are then mapped into the image domain, only the half cell, $(b)+(c)$ in Fig. 3 , needs to be treated instead of the whole cell $(a)+(b)+(c)+(d)$.

The Jacobian of the mapping function (eqn. 1) is

$$
\begin{aligned}
J & =\left|d z^{\prime} / d z\right|_{z=(x, y)}^{2} \\
& =a^{2}\left|\left[1-s^{2}(z, k)\right]\left[1-k^{2} s^{2}(z, k)\right]\right|
\end{aligned}
$$

From eqn. 2 it can be verified that

$$
J \rightarrow r^{\prime}
$$

near the strip edge, where $r^{\prime}$ is the distance from the strip's edge in the original domain. By using the relationship between electric potential and electric field intensity and imposing the Cauchy-Riemann's equations [17], it can be proved that the transverse electric field $\vec{E}_{t}^{\prime}$ in the original domain and the corresponding transverse field $\vec{E}_{t}$

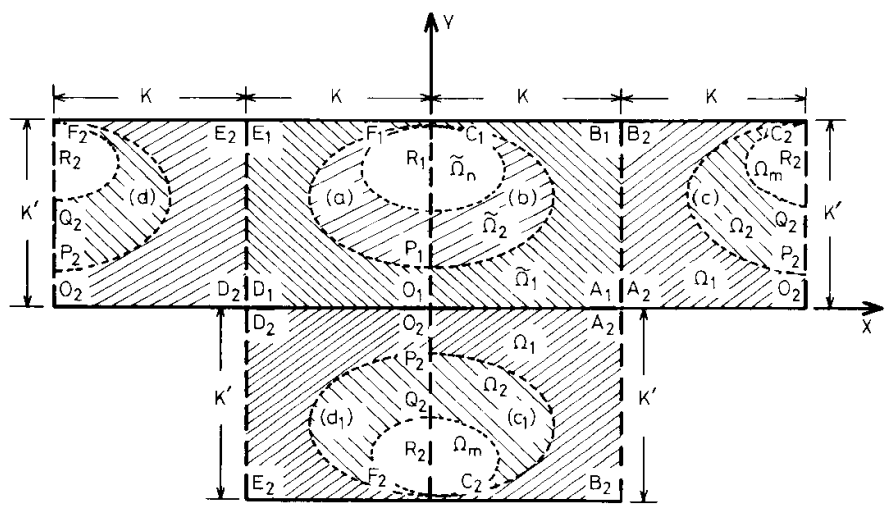

Fig. 2 Image domain corresponding to Fig. I the rectangular cell $(a)+(b)+(c)+(d))$ in $z$-plane $(z=x+j y)$ 
in the image domain are related by

$$
\vec{E}_{t}^{\prime}=\frac{\left|\vec{E}_{t}\right|}{\sqrt{ }(J)}[\cos (\Phi+\Psi) \hat{x}+\sin (\Phi+\Psi) \hat{y}]
$$

where $\Psi$ denotes the argument of the first derivative of the complex mapping function (eqn. 1), and $\Phi$ is the angle
Here, $\widetilde{\Omega}_{k}$ and $\Omega_{k}$ denote any of $\widetilde{\Omega}_{1}, \widetilde{\Omega}_{2}, \ldots, \Omega_{n}$ and $\Omega_{1}$, $\Omega_{2}, \ldots, \Omega_{m}$, respectively, and $\tilde{\varepsilon}_{k}$ and $\varepsilon_{k}$ denote any of $\tilde{\varepsilon}_{1}$, $\tilde{\varepsilon}_{2}, \ldots, \tilde{\varepsilon}_{n}$ and $\varepsilon_{1}, \varepsilon_{2}, \ldots, \varepsilon_{m}$, respectively. On the electric walls, $\left(\tilde{\Gamma}_{13}^{n}+\Gamma_{13}\right)$ and $\left(\tilde{\Gamma}_{12}+\Gamma_{12}\right)$, the essential boundary conditions $\phi=1$ and $\phi=0$ should be imposed. On the magnetic walls, $\tilde{\Gamma}_{11}$ and $\Gamma_{11}$, the essential boundary condition $\partial \phi / \partial n=0$ should also be enforced. In eqn. 6 ,

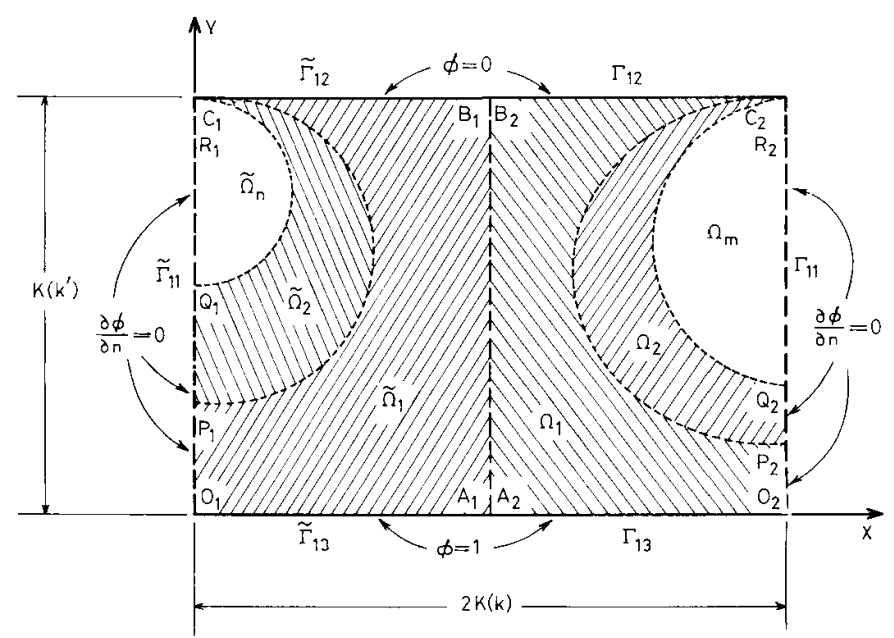

Fig. 3 Half cell $((b)+(c)$ of Fig. 2) for finite-element analysis and boundary conditions

defined by $\tan ^{-1}\left(E_{y} / E_{x}\right)$. The field in the image domain is then smooth everywhere, which makes the field in the original domain proportional to $1 / \sqrt{ }\left(r^{\prime}\right)$ and satisfy the required edge condition. Therefore, the field singularity difficulty in the original domain can be removed.

\subsection{Finite-Element Method}

Under quasi-static approximation, the potential $\phi$ in each homogeneous region of image domain (Fig. 3) satisfies the Laplace equation $\left(\nabla^{2} \phi=0\right)$. By multiplying this Laplace equation by a weighting function $w$, integrating the multiplied equation over a homogeneous region $\Omega_{j}$ (of constant permittivity $\varepsilon_{j}$ ) which is bounded by a closed curve $\Gamma_{j}$ and using Green's two-dimensional theorem in manipulation, the following equation may be obtained:

$$
\int_{\Omega_{j}}\left(\varepsilon_{j} \nabla w \cdot \nabla \phi\right) d \Omega=\int_{\Gamma_{j}} \varepsilon_{j} w \partial \phi / \partial n d \Gamma
$$

Applying eqn. 5 to each homogeneous region in the image domain (Fig. 3), and then suitably combining the resultant equations, the governing integral equation in the image domain may finally be obtained as

$$
\begin{aligned}
\sum_{k=1}^{n} \int_{\tilde{\Omega}_{k}}\left(\tilde{\varepsilon}_{k} \nabla w \cdot \nabla \phi\right) d \Omega+\sum_{k=1}^{m} \int_{\Omega_{k}}\left(\varepsilon_{k} \nabla w \cdot \nabla \phi\right) d \Omega \\
=\int_{\tilde{\Gamma}_{12}+\tilde{\Gamma}_{13}}\left(\tilde{\varepsilon}_{1} w \partial \phi / \partial n\right) d \Gamma \\
\quad+\int_{\tilde{\Gamma}_{12}+\tilde{\Gamma}_{13}}\left(\varepsilon_{1} w \partial \phi / \partial n\right) d \Gamma
\end{aligned}
$$

we have incorporated the constant $\tilde{\varepsilon}_{k}$ in the term associated with $\widetilde{\Omega}_{k}, \varepsilon_{k}$ in that with $\Omega_{k}, \tilde{\varepsilon}_{1}$ in that with $\left(\tilde{\Gamma}_{12}\right.$ $\left.+\tilde{\Gamma}_{13}\right)$ and $\varepsilon_{1}$ in that with $\Gamma_{12}+\Gamma_{13}$. By this choice, the continuity condition for the normal component of electric flux density may naturally be satisfied.

The integral equation (eqn. 6) is solved in the image domain by the Galerkin finite-element method [18]. In this study, the triangular elements as shown in Fig. 4 are

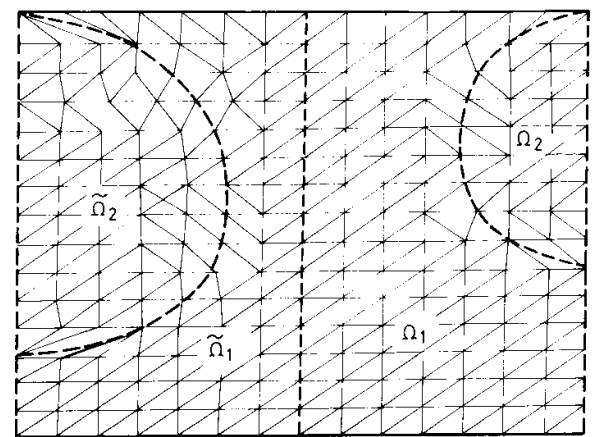

Fig. 4 Typical subdivision with triangular elements in image domain

adopted. The potential $\phi$ in each element is expressed as

$$
\phi=\sum_{j=1}^{3} \phi_{j} B_{j}
$$

where $B_{j}$ is a linear basis function and $\phi_{j}$ is the potential value at node $j$. The relationship between the linear basis 
functions and the global co-ordinate $(x, y)$ of a point in an element is given by [18]

$$
\left[\begin{array}{l}
B_{1} \\
B_{2} \\
B_{3}
\end{array}\right]=\left[\begin{array}{ccc}
x_{1} & x_{2} & x_{3} \\
y_{1} & y_{2} & y_{3} \\
1 & 1 & 1
\end{array}\right]^{-1}\left[\begin{array}{l}
x \\
y \\
1
\end{array}\right]
$$

where $\left(x_{i}, y_{i}\right), i=1,2,3$ are the global co-ordinates of the $i$ th vertex of triangular element. In Galerkin's method, the weighting function in each element is chosen as

$$
w=B_{i} \quad i=1,2,3
$$

By substituting eqns 7,8 and 9 into eqn. 6 , assembling the matrix for each element, the global matrix equation of the form

$$
[A][\phi]=[G]
$$

is obtained. Here $[\phi]$ is the column vector associated with the potential $\phi_{j}$ at each node, $[G]$ is the column vector due to the boundary terms and $[A]$ is a known sparse matrix. Although $[\phi]$ and $[G]$ both contain the nodal unknown $\phi_{j}$, eqn. 10 can still be solved because only one unknown is associated with each node.

\subsection{Effective dielectric constant and characteristic} impedance

From eqn. 10 the total charge $Q$ can be determined on signal strip from which the capacitance $C$ per unit length of coplanar line may be calculated

$$
C=Q / V=Q
$$

The effective dielectric constant $\varepsilon_{e f f}$ and characteristic impedance $Z_{0}$ can then be computed by [19]

$$
\begin{aligned}
& \varepsilon_{e f f}=C / C_{a} \\
& Z_{0}=\frac{\sqrt{ }\left(\varepsilon_{0} \mu_{0}\right)}{\sqrt{ }\left(C C_{a}\right)}
\end{aligned}
$$

where $C_{a}$ is the capacitance of the air-filled structure.

\section{Numerical results and discussions}

A computer software package has been developed on the VAX-11/780 computer, which also contains an automatic mesh division of triangular elements in the image domain. From the convergent test, it is found that a mesh division of 420 elements is usually enough to give results within four digit accuracy. A typical division in image domain is shown in Fig. 4.

To check the validity of this method, our calculated results for coplanar waveguide are presented in Fig. 5 and compared with those from the conformal-mapping method [9] and stationary formula [11]. It is found that all three methods give almost the same characteristic impedances for wide range of parameters. For effective dielectric constants, the present results agree well with previous results only in the larger $a / b$ range, implying that the previous conformal-mapping technique and stationary-formula approach are not adequate in the smaller $a / b$ range.

The effects of the single upper dielectric layer are presented in Figs. 6 and 7. The existence of the upper dielectric layer causes an increase of effective dielectric constant and a decrease of characteristic impedance. The increasing dielectric constant of the upper layer also has the same effect of increasing the layer thickness.

In Fig. 8, the effects of inserting two dielectric films, one on the top and the other on the bottom of the metallic electrodes, are presented. The addition of lower dielectric film on the conventional coplanar waveguide causes both an increase of characteristic impedance and a decrease of effective dielectric constant. Note also that the modification of both effective dielectric constant and characteristic impedance is not negligible even when inserting a thin dielectric film below the metallic electrodes.

The charge distributions over signal strip of coplanar line are shown in Fig. 9. Our numerical results exactly

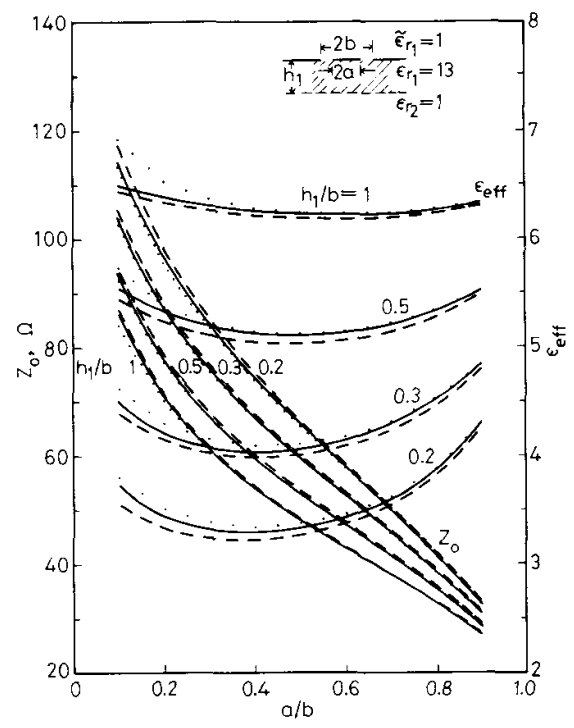

Fig. 5 Characteristic impedance $Z_{0}$ and effective dielectric constan

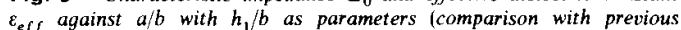
results)

present method conformal mapping

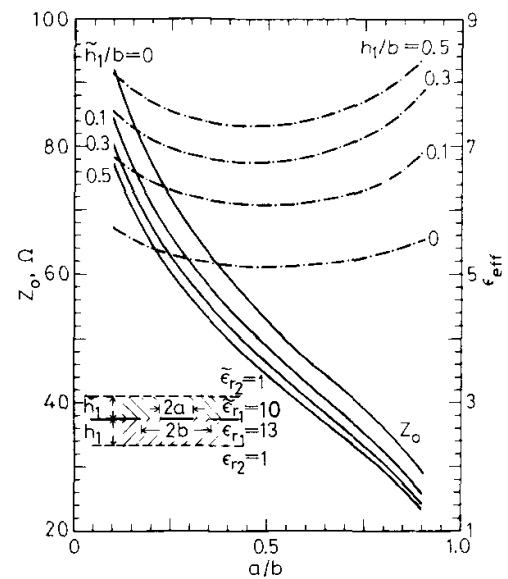

Fig. 6 Characteristic impedance $Z_{0}$ and effective dielectric constan $\varepsilon_{\text {eff }}$ against $a / b$ with $\bar{h}_{1} / b$ as parameters (effects of upper dielectric layer) $h_{1} b=0.5$

IEE PROCEEDINGS-H, Vol. 138 , No 4, AUGUST 1991 
reflect the singularity behaviour of charge distributions near conductor edges. Also observed in Fig. 9 is the decrease in charge distributions when the dielectric thickness is reduced.

The tangential field distributions over the slots of the coplanar line are shown in Figs. 10 and 11. These results again reflect the singularity behaviour of electric fields near conductor edges. The tangential field distributions (Fig. 10) are nearly symmetric over the slots and nearly independent of the dielectric thickness, which supports the assumption of the spectral-domain method [20]. Fig. 11 reveals that stronger fields gather between metallic electrodes as dielectric constants of films are increased, which is a consequence of more field confinement caused by a greater dielectric constant

In the previous studies, the magnetic wall assumption over slots was made so that a closed-form solution could be obtained by the conformal-mapping analyses [4-10]

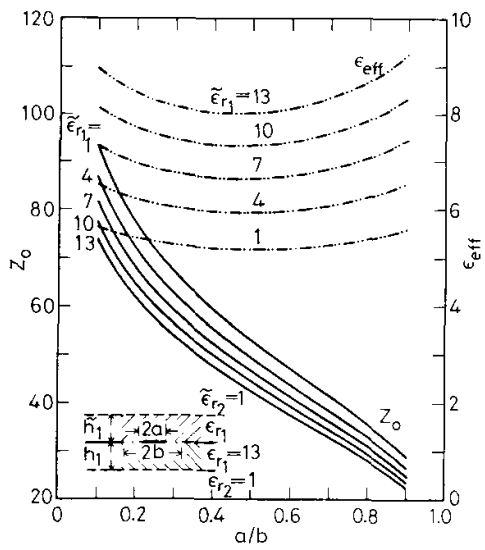

Fig. 7 Characteristic impedance $Z_{0}$ and effective dielectric constant $\varepsilon_{\text {efs }}$ against $a / b$ with $\bar{\varepsilon}_{\mathrm{r} 1}$ as parameters. $h_{1} / b=0.5, \tilde{h}_{1} / h_{1}=0.5$ (effects of upper dielectric layer

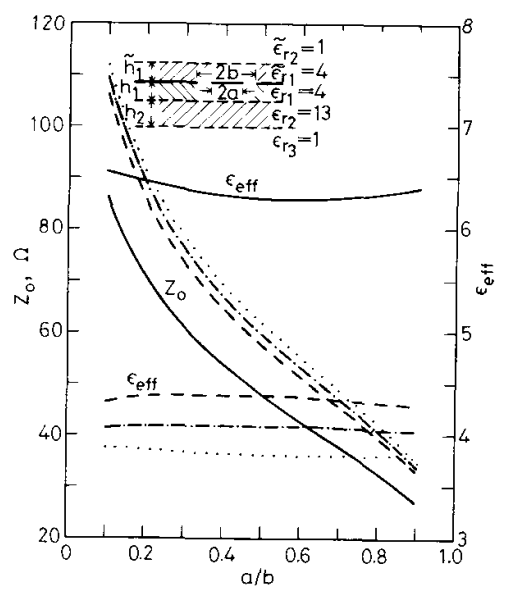

Fig. 8 Characteristic impedance $Z_{0}$ and effective dielectric constan $\varepsilon_{\text {efs }}$ against $a / b$ with $h_{1} / b$ as parameters. $\tilde{h}_{1} / h_{1}=1, h_{1}+h_{2}=b$ (effects of upper and lower dielectric layers)

of upper and lower

$h_{1} / b=0$
$h_{1} b=0.1$

$h_{1} / b=0.2$
$h_{1} / b=0.5$

IEE PROCEEDINGS-H, Vol. I38, No. 4, AUGUST 1991
The validity of this assumption is tested in this study. Shown in Fig. 12 are the ratios of $E_{y} / E_{x}$ for various substrate thicknesses. The magnetic wall assumption is acceptable only when the ratio $h_{1} / b$ is greater than some specific number such as two in this special case.

\section{Conciusions}

A novel hybrid approach that combines the Galerkin finite-element method and the conformal-mapping technique has been presented for a quasistatic analysis of multilayer coplanar lines. This approach, without using singular elements and infinite elements, is characterised by its ease in handling both field singularity behaviour and the infinite extension of field. Numerical results for

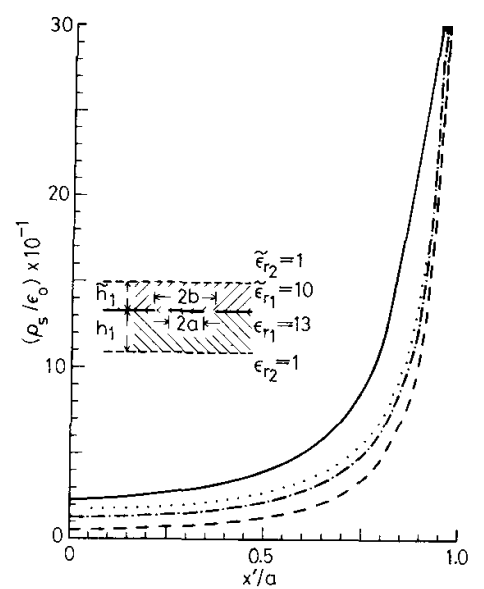

Fig. 9 Charge distribution over signal strip with $h_{1} / b$ as parameters. $a / b=0.5, \tilde{h}_{1} / h_{1}=0.5$

$h_{1} / b=0.2$

$h_{1} / b=0.4$

$h_{1} / b=0.6$

$h_{1} / b=1.0$

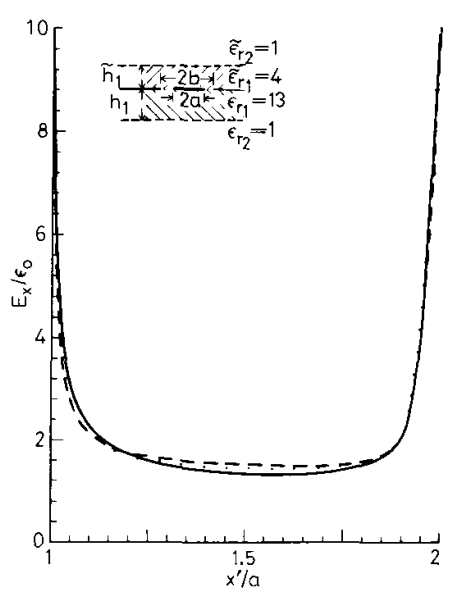

Fig. 10 Tangential field distribution over slot with $h_{1} / b$ as parameters $a / b=0.5$
$h_{2} / b=0.1$

$--h_{1} / b=0.2$

$h_{1} / b=0.5$ 
effective dielectric constants, characteristic impedances, charge distributions and field distributions over slots were shown and compared with the available literature. The magnetic wall assumption of previous studies has also been carefully examined.

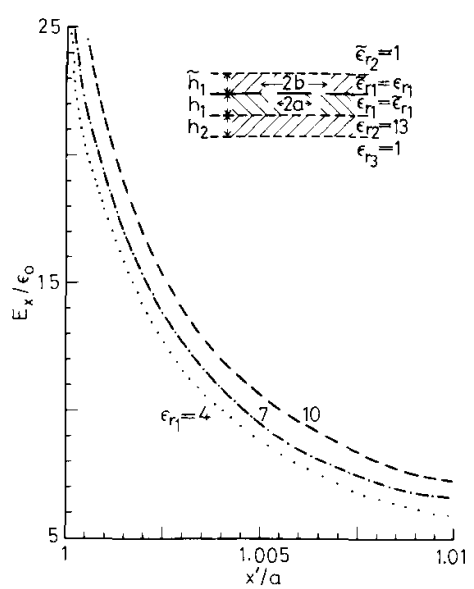

Fig. 11 Tangential field distribution over slot of $\varepsilon_{r_{1}}$ as parameters $a / b=0.5$ $h_{2} / b=0.9$
$h_{1} / b=\mathscr{h}_{1} / b=0.1$

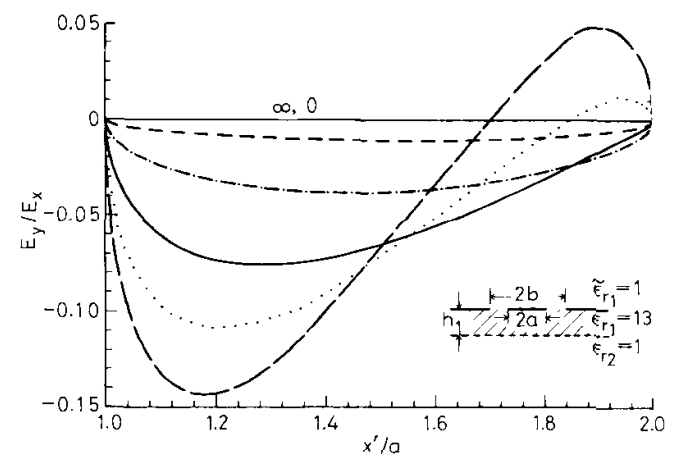

Fig. 12 Ratio of normal $E\left(E_{y}\right)$ to tangential $E\left(E_{x}\right)$ over slot. $a / b=0.5$

$h_{1} / b=0.2$

$H / b=0.3$

$h_{1} / h=0$
$h_{1} / h=1$

$h_{1} / b=1.0$
$h_{1} / b=2.0$

\section{Acknowledgment}

This work was supported by the National Science Council, Republic of China, under grant NSC 78-0404E002-06.

\section{References}

1 MURAGACHI, MT HIROTA T., MINAKAWA, A., OHWADA K., and SUGETA, T.: 'Uniplanar MMIC's and their applications', IEEE Trans., 1988, MTT-36, (12), pp. 1896-1900

2 1TOH, T.: 'Overview of quasi-planar transmission lines', IEEE Trans., 1989, MTT-37, (2), pp. 275-280

3 RIAZIAT, M., MAJIDI-AHY, R., and FENG, I.J.: 'Propagation modes and dispersion characteristics of coplanar waveguides', IEEE Trans., 1990, MTT-38, (3), pp. 245-251

4 WEN, C.P.: 'Coplanar waveguide: a surface strip transmission line suitable for nonreciprocal gyromagnetic device applications', IEEE Trans., 1969, MTT-17, (12), pp. 1087-1090

5 DAVIS, M.E., WILLIAMS, E.W., and CELESTINI, A.C.: 'Finiteboundary corrections to the coplanar waveguide analysis', IEEE Trans. 1973, MTT-21, (9), pp. 594-596

6 VEYRES, C., and FOUAD HANNA, V.: 'Extension of the application of conformal mapping techniques to coplanar lines with finite dimensions', Int. J. Electron., 1980, 48, (1), pp. 47-56

7 FOUAD HANNA, $V$, and THEBAULT, D.: 'Analysis of asymmetrical coplanar waveguides', Int. J. Electron., 1981, 50, (3), pp. 221-224

8 SHIH, Y.C., and ITOH, T.: 'Analysis of conductor backed coplanar waveguide', Electron. Lett., 1982, 18, (12), pp. 538-540

9 FOUAD HANNA, V., and THEBAULT, V.: 'Theoretical and experimental investigation of asymmetric coplanar waveguide', IEEE Trans., 1984, MTT-32, (12), pp. 1649-1651

10 GHIONE, G., and NALDI, C.U.: 'Coplanar waveguides for MMIC applications: effect of upper shielding, conductor backing, finiteextent ground planes, and line-to-line coupling', IEEE Trans., 1987 extent ground planes, and

11 KITAZAWA, T., and HAYASHI, Y.: 'Coupled slots on an anisotropic sapphire substrate', IEEE Trans., 1981, MTT-29, (10), pp. $1035-1040$

12 BOIX, R.R., and HORNO, M.: 'Modal quasistatic parameters for coplanar multiconductor structures in multilayered substrates with arbitrary transverse dielectric anisotropy', IEE Proc. $H$, Microwaves, Antenna \& Propag., 1989, 136, (1), pp. 76-79

13 HATSUDA, T.: 'Computation of coplanar-type strip-line characteristics by relaxation method and its application to microwave ciristics by relaxation method and its application to
cuits', IEEE Trans., 1975, MTT-23, (8), pp. 795-802

14 PANTIC, Z., AND MITTRA, R.: 'Quasi-TEM analysis of microwave transmission lines by the finite-element method', IEEE Trans., 1986, MTT-34, (11), pp. 1096-1103

15 GRADSHTEYN, I.S., and RYZHIK, I.M.: 'Table of integrals, series, and products' (Academic Press, New York, 1965)

16 MATHEWS, F., and WALKER, R.L.: 'Mathematical methods of physics' (W.A. Benjamic Inc., New York, 1970)

17 CHURCHILL, R.V., and BROWN, J.W.: 'Complex variables and applications' (Mc-Graw-Hill, New York, 1984)

18 ZIENKIEWICZ, O.C.: 'The finite element method' (McGraw-Hill, New York, 1977)

19 GUPTA, K.C., GARG, R., and BAHL, I.J.: 'Microstrip lines and slotlines' (Dedham, MA: Artech House Inc., 1979)

20 KNORR, J.B., and KUCHLER, K.D.: 'Analysis of coupled slots and coplanar strips on dielectric substrate', IEEE Trans., 1975, MTT-23, (7), pp. 541-548 